\title{
OPTIMUM CAPACITY ALLOCATION OF DISTRIBUTED GENERATION UNITS USING PARALLEL PSO USING MESSAGE PASSING INTERFACE
}

\author{
Rosamma Thomas $^{1}$, Jino M Pattery $^{2}$, Surumi Hassainar ${ }^{3}$ \\ ${ }^{1}$ M.Tech Student, Electrical and Electronics, FISAT, Kerala, India, rosamma.tk@gmail.com \\ ${ }^{2}$ Senior Engineer, Product Division, Kalkitech, Kerala, India, jino.pattery@kalkitech.in \\ ${ }^{3}$ Assistant Professor, Electrical and Electronics, FISAT, Kerala, India, surumishf@gmail.com
}

\begin{abstract}
This paper proposes the application of Parallel Particle Swarm Optimization (PPSO) technique to find the optimal sizing of multiple $D G($ Distributed Generation) units in the radial distribution network by reduction in real power losses and enhancement in voltage profile. Message passing interface (MPI) is used for the parallelization of PSO. The initial population of PSO algorithm has been divided between the processors at run time. The proposed technique is tested on standard 123-bus test system and the obtained results show that the simulation time is significantly reduced and is concluded that parallelization helps in enhancing the performance of basic PSO. The procedure has been implemented in an environment in which OpenDSS (Open Distribution System Simulator) is driven from MATLAB. An adaptive weight particle swarm optimization algorithm has been developed in MATLAB , parallelization is achieved using MATLABMPI and the unbalanced three-phase distribution load flow (DLF) has been performed using Electric Power Research Institute's (EPRI) open source tool OpenDSS.
\end{abstract}

Index Terms: Distributed Generation, Message Passing Interface, Optimal Placement, Parallel Particle Swarm Optimisation

\section{INTRODUCTION}

Distribution network characteristics include radial structure, unbalanced operation, high $\mathrm{R} / \mathrm{X}$ ratios, and nowadays distributed generation(DG) too . Distribution systems were designed to operate under unidirectional power flow conditions, but with distributed generation in the network the power flow is no longer unidirectional [1]. Some benefits of installation of distributed generation to the distribution system are improving voltage profile, reduction in losses, and providing backup power [2]. Reverse power flow may occur and can cause voltage rise problem, if penetration level is too high and also the power loss in the network may be more due to inappropriate sizing of DG than that of the case without any DG [3] , [4]. Deep and detailed studies are needed for the analysis of the distribution network with DG. For these studies to be possible an appropriate power flow algorithm is necessary. So the distribution load flow is carried out using OpenDSS (Open Distribution System Simulator).

The global minimum is easily found out by particle swarm optimization (PSO) algorithm. It can be used to optimize objective functions which are not differentiable and irregular. So PSO based unbalanced three-phase power flow can be used to solve DG capacity problem in a distribution network [5]. But it has the disadvantage that, since the algorithm simulates the movement of a swarm of particle solutions over a very large number of iterations, it takes a significant processing time. So a parallel implementation of the PSO is proposed to reduce the computation time. In this approach the basic PSO with a very large number of particles and iterations are simulated in a reduced time[6].

The paper has been organised as follows: In section 2, PSO algorithm is described briefly. Section 3 describes parallel PSO using MPI. The objective function formulation of DG generation capacity is explained in section 4.1. Implementation of the procedure is given in section 4.2 . Test system and results are given in section 5 .

\section{PARTICle SWARM OPTIMIZATION}

Kennedy and Eberhart proposed Particle swarm optimization (PSO) algorithm which is a population-based optimization method inspired by social behavior of bird flocking or fish schooling[7]. In this method individuals called particles change their position during the optimization process. Each particle adjusts its position according to its own experience (pbest), and according to the experience of a neighboring particle (gbest). The velocity at which the particles move is given by the equation (1).

$v_{i}^{k+1}=w v_{i}^{k}+c_{1} r_{1}\left(\right.$ pbest $\left._{i}-s_{i}^{k}\right)+c_{2} r_{2}\left(\right.$ gbest $\left._{i}-s_{i}^{k}\right)$

The particle's new position in the search space is given by 
the equation(2).

$$
s_{i}{ }^{k+1}=s_{i}^{k}+v_{i}^{k+1} \quad i=1,2, \ldots, n
$$

where,

$s^{k}$ is current searching point,

$s^{k+1}$ is modified searching point,

$v^{k+1}$ is modified velocity of agent $\mathrm{i}$,

$n$ is number of particles in a group,

pbest $_{i}$ is pbest of agent $i$,

gbest $_{i}$ is gbest of the group,

$w$ is weight function for velocity of agent $\mathrm{i}$,

$r_{1}$ and $r_{2}$ are random numbers between 0 and 1

$c_{i}$ is weight coefficients for each term.

Inertia weight can be calculated by equation (3) .

$$
w_{i}=w_{\max }-\left\{\left[\left(w_{\max }-w_{\min }\right) k\right] / k_{\max }\right\}
$$

where,

$w_{\min }$ and $w_{\max }$ are the minimum and maximum weights respectively. $k$ and $k_{\max }$ are the current and maximum iteration. Appropriate value ranges for $c_{1}$ and $c_{2}$ are between 1 and 2 .

\section{PARALLEL PSO USING MPI}

To analyze distribution systems of realistic size, some refinements are needed to significantly reduce the simulation time of basic PSO. The efficiency of PSO algorithm can be improved largely by parallel computing technique. Multiple processors are used to implement the algorithm which reduces the simulation time. Hence complex functions can be optimized using parallel PSO [11]. MPI is used to parallelise PSO so easily. In this paper the swarm particles of basic PSO are distributed equally among processors such that all processors have equal load.

\section{OPTIMUM CAPACITY ALLOCATION OF DG UNITS BASED ON PARALLEL PSO}

\subsection{Objective Function Formulation}

The objective of the paper is to determine the optimum generation capacity of multiple DG units in an unbalanced distribution network so that efficiency is enhanced and stability is ensured. The utility's operating cost can be reduced and efficiency can be enhanced by reducing the power losses of the distribution area. So, objective function is considered as follow:

$f_{o b j}=\sum_{k=1}^{n} P_{L}(k)$

where, $P L$ is the power loss in power delivery lines and $n$ is the total number of distribution lines[5].
Reverse power flow may occur if DG penetration is too high and this causes over voltage at the buses[3]. Therefore, steady-state voltage stability limits have been considered as inequality constraint. If $v_{\min }$ and $v_{\max }$ are the minimum and maximum voltage limit and $N$ is the total number of nodes, then voltage stability limit can be written as:

$$
v_{\min }<v_{i}<v_{\max }, \quad i \forall N
$$

In this analysis, the inequality constraint is added to the objective function using penalty factors[6]. Therefore, overall objective function can be written as:

$$
\min f(x, u)
$$

where

$$
f(x, u)=f_{o b j}(x, u)+f_{p}(x, u)
$$

subject to

$$
g(x, u)=0
$$

with

$$
x \in R^{n} \text { and } u \in R^{m}
$$

Three different types of DG technologies have been considered for optimum capacity allocation[5].

They are:

- Type-1 DG: which delivers real power only; therefore, power factor is 1. e.g. PV cell, fuel cells etc.

- Type-2 DG: which delivers both real and reactive power, therefore, power factor is positive (In this analysis $\mathrm{pf}=0.88$ has been considered). e.g. synchronous generators.

- Type-3 DG: which delivers real power but consumes reactive power, therefore, power factor is negative (In this analysis $\mathrm{pf}=-0.88$ has been considered).e.g. induction generators

\subsection{Implementation of the Procedure}

Optimum generation capacity of multiple DG units are found out using PSO algorithm implemented in Matlab. Unbalanced three phase DLF is done using OpenDSS. At every iteration Matlab and OpenDSS interact with each other. OpenDSS has a COM (Component Object Model) server DLL (Dynamic-link library) that can be driven from other software platforms such as Matlab[10]. For parallelization of PSO MatlabMPI is used. It is set of Matlab scripts that implement a subset of MPI and allow any Matlab program to be run on a parallel computer. MatlabMPI will run on any combination of computers that Matlab supports[10].The block diagram of the implemented procedure is shown in Fig. 2 and flowchart is given in Fig. 1 


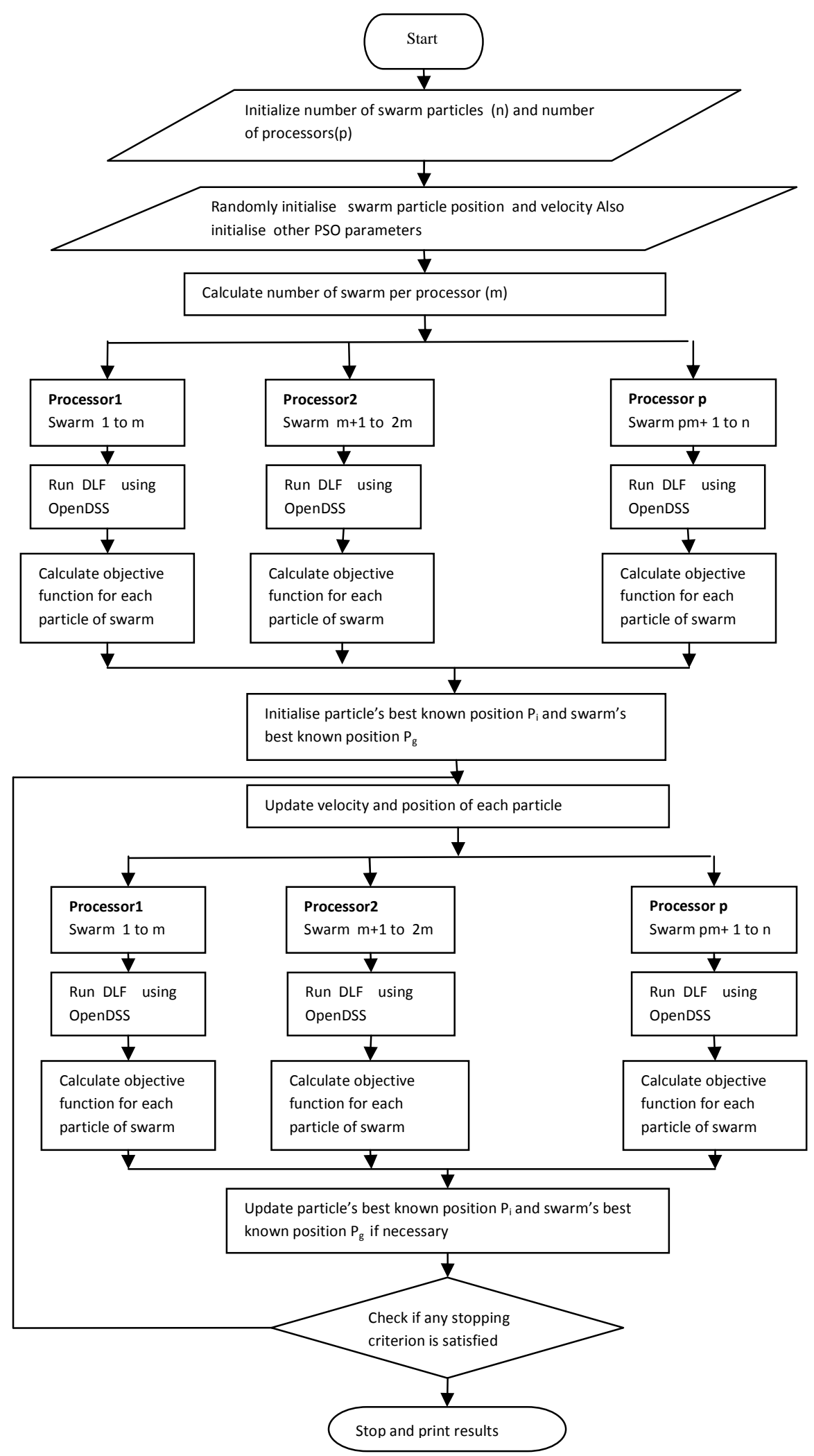

Fig.1 Flowchart of the implemented procedure 


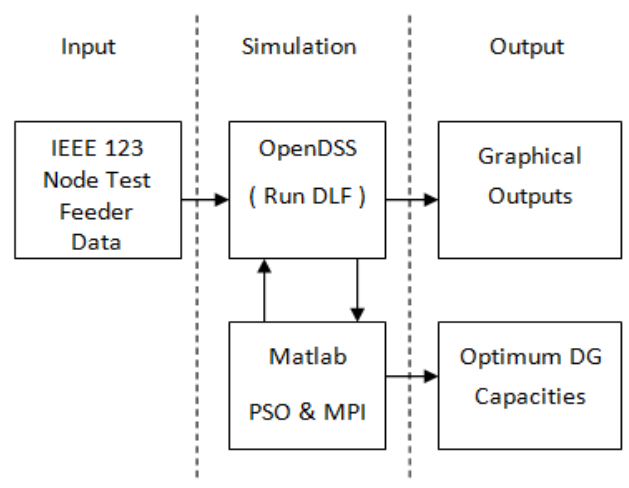

Fig. 2 Block diagram of implemented procedure

\section{TEST SYSTEM AND RESULTS}

The analysis has been carried out on IEEE 123 node test distribution feeder which is three-phase unbalanced in nature. The IEEE 123 node test system does not have any DG in the base case. In this analysis potential sites for DG installation have been selected prior to the analysis.

In [5] the author has used basic PSO for optimising multiple DG unit capacities. The data used in [5] are as follows. In [5] DG units have been installed in the heavily loaded buses selected which are bus 76 , bus 48 and bus 65 . Optimal generation capacity of DG units installed in those buses has been determined. For the optimization process each DG capacity has been limited by the equation(9).

$$
P_{D G}^{\min }<P_{D G}^{i}<P_{D G}^{\max }, \quad i \forall N
$$

where $P_{D G}^{\min }$ is $90 \mathrm{~kW}$ (around 2.5\% penetration) and $P_{D G}^{\max }$ is $3 \mathrm{MW}$ (around $85 \%$ penetration) and $N$ represents the number of DG units. The same case is simulated with parallel PSO in this paper.

The purpose of this test scenario is to analyze computational complexity and time and to examine the impact of multiple optimum sized DG on power loss profile and voltage profile of the network. As the number of DG units increases and the network size increases, solution complexitywill increase to determine the optimum generation capacity. Completeness and speed are two qualities of the proposed method.

In this work the computations are performed on a HPDL980G8 hardware with 4 processor each with 10 cores. The simulation is conducted in a virtual environment with four operating system with one master node and three slave nodes.

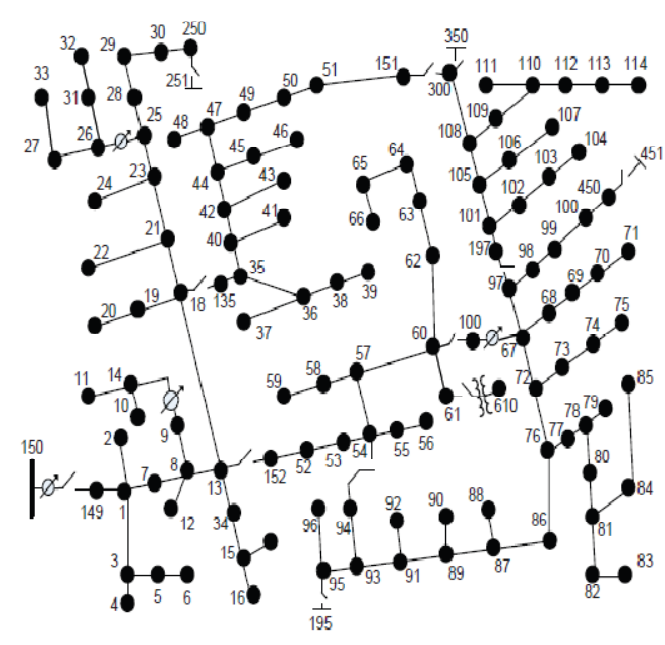

Fig.3 IEEE node test feeder

Results for optimum DG capacity at bus 76,48 and 65 using the proposed method of parallel PSO using MPI for three different DG technologies are given in Table I.

Table 1

Comparison Of Solution Time With Basic PSO And Parallel PSO

\begin{tabular}{|l|l|l|l|l|}
\cline { 3 - 5 } \multicolumn{2}{c|}{} & \multicolumn{3}{l|}{ DG Technology } \\
\cline { 2 - 5 } \multicolumn{2}{l|}{} & $\begin{array}{l}\text { Type-1 } \\
\text { DG }\end{array}$ & $\begin{array}{l}\text { Type-2 } \\
\text { DG }\end{array}$ & $\begin{array}{l}\text { Type-3 } \\
\text { DG }\end{array}$ \\
\hline \begin{tabular}{|l|l|l|} 
Optimum \\
DG \\
Dapacity \\
(MW)
\end{tabular} & at bus 76 & 1.58 & 1.22 & 1.21 \\
\cline { 2 - 5 } & at bus 48 & 0.99 & 1.02 & 0.52 \\
\hline $\begin{array}{l}\text { Solution time with } \\
\text { basic PSO (s) }\end{array}$ & 0.39 & 0.39 & 0.20 \\
\hline $\begin{array}{l}\text { Solution time with } \\
\text { parallel PSO (s) }\end{array}$ & 6.91 & 19.90 & 21.30 \\
\hline Power Loss (kW) & 20.17 & 19.30 & 51.40 \\
\hline $\begin{array}{l}\text { Power Loss } \\
\text { Reduction (PLR)(\%) }\end{array}$ & 78.94 & 79.80 & 46.34 \\
\hline
\end{tabular}

Installation of multiple DG units with optimum capacity will decrease the network power loss significantly. Power loss of the network becomes $20.17 \mathrm{~kW}, 19.3 \mathrm{~kW}$ and $51.4 \mathrm{~kW}$ respectively for type-1, type-2 and type-3 optimum sized DG units. Power loss of 123 node test feeder without any DG is $95.8 \mathrm{~kW}$. Power loss reduction(PLR) by the DG units can be calculated using (10) [5]. Power loss reduction is less for type-3 DG units as they consume reactive power from the 
grid; therefore, more power loss occurs compared with the other two DG technologies[5].

$$
P L R=\left(\left(P_{L O S S}-P_{L O S S}^{D G}\right) / P_{L O S S}\right) * 100 \%
$$

where,

$P_{\text {LOSS }}=$ Power loss of the system before introducing DG

$P_{L O S S}^{D G}=$ Power loss of the system after adding DG

From the table it is clear that with parallelisation of PSO the simulation time is reduced compared to simulation with basic PSO used in [5].Following figure shows the location of three type-1 DG units.

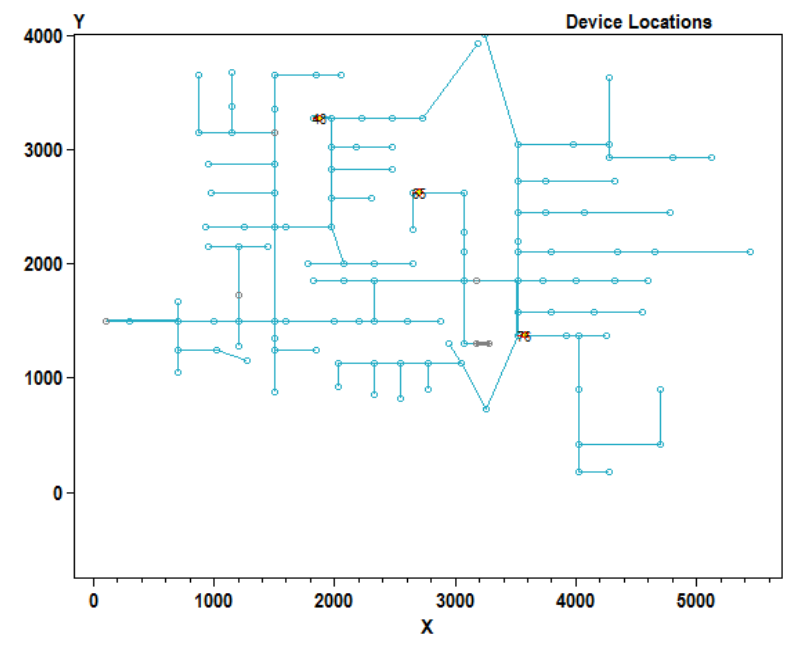

Fig. 4 Location of 3 Type1 DG units in the network

From Fig. 5 it is observed that the steady state voltage at all nodes are within the permissible limit of $0.94 \mathrm{pu}$ and $1.06 \mathrm{pu}$. Thus by optimization of the DG capacities the voltage profile is also improved.

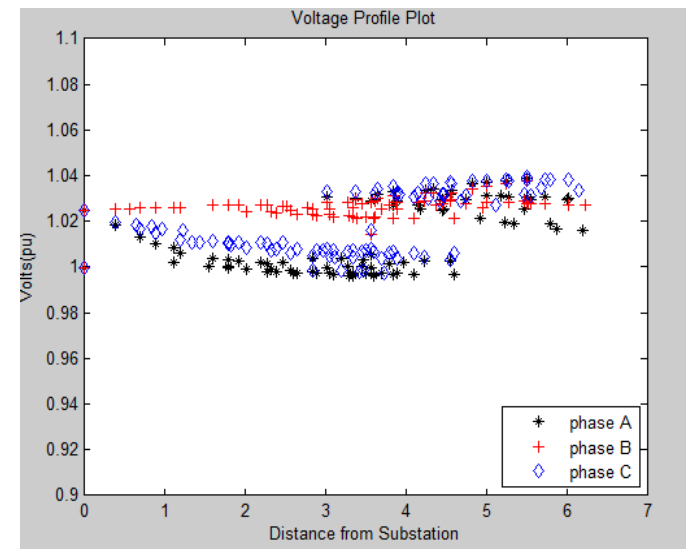

Fig. 5 Node Voltage considering 3 Type1 DG units

\section{CONCluSion}

From the results it can be easily concluded that, using multiple DG units with optimum generation capacity, power loss of network is reduced and voltage profile remains within stability margin. Also without the addition of any extra parameter PSO can be parallelized which reduces the simulation time. A major drawback in parallel programming is communication between processors which cannot be removed completely. So for getting a considerable reduction in time with multi processors the problem size should be large. Distribution system networks are fairly large in size, so this method can be used to optimize distribution system problems.

\section{REFERENCES}

[1] V. Janev. Implementation and evaluation of a distribution load flow algorithm for networks with distributed generation. Bachelor's Thesis, ETH Zurich, 2009

[2] H. Lee Willis and W.G. Scott, Distributed Power Generation. Planning and Evaluation, Marcel Dekker, 2000.

[3] P. Carvalho, P. Correia, and L. Ferreira, "Distributed reactive power generation control for voltage rise mitigation in distribution networks," IEEE Transactions on Power Systems, vol. 23, no. 2, pp. 766-772, May 2008.

[4] V. Quezada, J. Abbad, and T. Roman, "Assessment of energy distribution losses for increasing penetration of distributed generation," IEEE Transactions on Power Systems, vol. 21, no. 2, pp. 533-540, May 2006.

[5] Adnan Anwar and H. R. Pota, 'Optimum Capacity Allocation of DG Units based on Unbalanced Three-phase Optimal Power Flow' in IEEE PES General Meeting, San Diego, USA, Jul. 2012.

[6]V. Roberge and M. Tarbouchi"Parallel particle swarm optimization on graphical processing unit for pose estimation”. WSEAS Transactions on Computers , 11(6):170 179, June 2012.

[7] J. Kennedy, and R. Eberhart, "Particle swarm optimization", IEEE Int. Conf. on Neural Networks IV, p. 1941-1948, Piscataway, NJ, 1995

[8] Eberhart and Y. Shi, "Particle swarm optimization: developments, applications and resources," in Proceedings of the 2001 Congress on Evolutionary Computation.

[9] "OpenDSS manual and reference guide." [Online]. Available: http://sourceforge.net/projects/electricdss

[10] "MatlamMPI reference guide .’[Online].Available: http://www.ll.mit.edu/mission/cybersec/softwaretools/matlab mpi/matlabmpi.html

[11] G.Singal, A. Jain and A. Patnaik, "Parallelization of Particle Swarm Optimization using Message Passing Interfaces" in NaBIC 2009 World Congress 